\title{
Rôle de l'apprentissage dans la gouvernance territoriale. Le cas d'une indication géographique en Algérie
}

The role of training in territorial governance: Geographical indication in Algeria

Sihem Ait Hammou, Fatiha Fort, Fatima Brabez et Rym Saidoun

\section{(2) OpenEdition}

Journals

Édition électronique

URL : https://journals.openedition.org/economierurale/7215

DOI : 10.4000/economierurale.7215

ISSN : 2105-2581

Éditeur

Société Française d'Économie Rurale (SFER)

Édition imprimée

Date de publication : 31 décembre 2019

Pagination : 47-60

ISSN : 0013-0559

\section{Référence électronique}

Sihem Ait Hammou, Fatiha Fort, Fatima Brabez et Rym Saidoun, « Rôle de l'apprentissage dans la gouvernance territoriale. Le cas d'une indication géographique en Algérie », Économie rurale [En ligne], 370 | octobre-décembre, mis en ligne le 01 janvier 2022, consulté le 06 janvier 2022. URL : http:// journals.openedition.org/economierurale/7215; DOI : https://doi.org/10.4000/economierurale.7215 


\title{
Rôle de l'apprentissage dans la gouvernance territoriale Le cas d'une indication géographique en Algérie
}

\author{
Sihem AIT HAMMOU • ENSA, CREAD, Alger, Algérie \\ Sihem_ait@yahoo.fr \\ Fatiha FORT • Université Montpellier, SupAgro, Montpellier \\ fatiha.fort@supagro.fr \\ Fatima BRABEZ, Rym SAIDOUN • ENSA, CREAD, Alger, Algérie \\ f.brabez@ensa.dz; rymel66@yahoo.fr
}

L'analyse de la gouvernance territoriale est présentée à travers l'élaboration du dispositif de reconnaissance de la qualité des produits agricoles et d'origine agricole. Cet instrument est mis en oeuvre en invitant les acteurs privés et associatifs à se joindre aux acteurs publics pour la définition des signes de qualité. L'article appréhende la gouvernance à travers les processus d'apprentissage collectif pour la reconnaissance de la qualité des produits d'origine. L'analyse de la gouvernance territoriale met en évidence des situations d'apprentissage de la participation et de la concertation induites par la mise en place de l'Indication géographique figue sèche de Béni Maouche.

MOTS-CLÉS : gouvernance territoriale, apprentissage, indication géographique, action collective, concertation

\section{The role of training in territorial governance: Geographical indication in Algeria}

This analysis of territorial governance is presented through the creation of the quality recognition system for agricultural products and products of agricultural origin. This instrument is implemented by inviting private and associative stakeholders to join public actors in order to determine the signs of quality. This article understands governance through the processes of collective training for recognizing the quality of products of agricultural origin. This analysis of territorial governance highlights the training on participation and coordination that emerged as a result of the establishment of the geographical indication label for the "dried figs of Béni Maouche." (JEL: 018, Q18).

KEYWORDS: territorial governance, training, geographical indication, collective action, cooperation

es différentes politiques agricoles qui ఒse sont succédé en Algérie depuis son indépendance ont vu s'appliquer des programmes conçus et planifiés le plus souvent de façon centralisée. En effet, dès les années post-indépendance, les pouvoirs publics se sont engagés dans des actions mises en œuvre de manière descendante. Le regroupement des fermes coloniales en domaines autogérés illustre bien cette logique. Les années 1970 ont vu se pérenniser ces pratiques. Avec la révolution agraire, une politique centralisée accorde un rôle primordial à l'État dans la conception et la formation de la politique.

Bien qu'il y ait eu des tentatives de libéralisation et de privatisation du mode de gestion de l'exploitation agricole, ce n'est que dans les années 2000 que les pouvoirs publics ont reconsidéré les politiques de développement, en réorientant l'action publique vers de nouveaux modes 
de gouvernance. Une nouvelle réforme de l'action publique se dessine alors, axée sur les notions de partenariat et de participation. Les pouvoirs publics lancent en 2009 la politique du renouveau agricole et rural (désignée plus loin sous l'expression abrégée «politique du renouveau agricole»), un ensemble d'actions ancrées dans une approche de développement territorial qui met l'accent sur la décentralisation et la responsabilisation des acteurs au niveau local.

Le concept de gouvernance a été appliqué au territoire par les travaux de Gilly et Wallet (2001) et Simoulin (2007). Pour Gilly et Wallet (2001), la gouvernance locale ou territoriale peut être considérée comme « un processus de confrontation et d'ajustement simultanés du système de représentation et des actions de groupes d'acteurs géographiquement proches mais éventuellement de domaines organisationnels différents afin de compléter un projet de développement local ». Cette définition met l'accent sur les processus sousjacents de confrontation et d'ajustement des différents acteurs de la gouvernance territoriale : institutionnels, économiques et sociaux. Ces différents acteurs doivent coopérer pour développer des formes de gouvernance territoriale, comme le suggère Simoulin (2007) : « La gouvernance territoriale couvre toutes les situations de coopération non ordonnées par la hiérarchie, correspondant à la construction, à la gestion ou à la représentation des régions, notamment en ce qui concerne leur environnement économique et institutionnel. »

Rey-Valette et Mathé (2012) ont proposé plus récemment un cadre méthodologique pour l'évaluation de la gouvernance territoriale où l'apprentissage est considéré comme un processus d'évaluation participative. Dans cet article, nous considérons l'apprentissage de l'action collective comme un processus permettant de construire la gouvernance territoriale. En effet, le projet de reconnaissance de la qualité liée au territoire des produits, déployé dans le cadre de la politique du renouveau agricole, constitue une opportunité pour l'apprentissage de l'action collective des acteurs engagés pour la construction de la gouvernance territoriale. Nous proposons d'analyser ces processus d'apprentissage à travers les évolutions institutionnelles et organisationnelles produites lors de la construction et de la mise en place de l'Indication géographique (IG) «figue sèche de Béni Maouche ».

Notre article est organisé comme suit : la première partie situe l'approche territoriale du dispositif de reconnaissance de la qualité au sein de l'ensemble plus large de la politique du renouveau agricole et rural. Dans la deuxième partie, une revue de la littérature permet d'appréhender la gouvernance territoriale à travers la question de l'apprentissage. La troisième partie est consacrée à l'étude du terrain algérien et au cas de la certification de la figue sèche de Béni Maouche par une IG. Enfin, nous proposons une analyse de la gouvernance territoriale sur la base de plusieurs situations d'apprentissage. Les résultats saillants et les perspectives d'approfondissement sont discutés dans la conclusion générale.

\section{La politique de valorisation des produits agricoles en Algérie}

La politique du renouveau agricole a pour ambition une autre manière de gouverner le secteur agricole et rural en Algérie. Fondée sur une responsabilité partagée et un partenariat entre les acteurs publics, privés et associatifs, elle intègre la dimension participative dans ses objectifs fondamentaux en favorisant l'adhésion des parties prenantes au développement, qui devient la préoccupation de l'ensemble des acteurs. Cette nouvelle manière de penser le développement dépasse la sphère des acteurs institutionnels en charge de la politique et des acteurs producteurs, pour s'étendre à 
d'autres parties prenantes au niveau du territoire : collectivités locales, acteurs privés et associations.

Le lien des produits au territoire est particulièrement mis en avant dans ce cadre. La loi d'orientation agricole de $2008^{1}$ introduit en effet la reconnaissance de la qualité liée au territoire. Ces dispositions posent un premier cadre réglementaire pour un système de reconnaissance de la qualité des produits agricoles ou d'origine agricole. Le lien au terroir, comme garantie de la qualité, est placé au centre de ce système, qui instaure ainsi les conditions de production et de certification de la qualité du produit, conditions pour la valorisation et la promotion des modes de production et des savoir-faire locaux. À la suite de cette réforme législative, un décret ${ }^{2}$ définit en 2013 les quatre signes distinctifs qui permettent la reconnaissance de la qualité, à savoir l'appellation d'origine, l'indication géographique, l'agriculture biologique et le label agricole de qualité.

\section{Le système national de labellisation : un instrument d'action publique}

Le système national de labellisation est organisé par le cadre réglementaire, comme un dispositif de reconnaissance de la qualité. Il s'agit d'une structure constituée d'un Comité national de labellisation, d'un secrétariat permanent et de deux autres entités : les sous-comités spécialisés et les organismes de certification. Le Comité de labellisation regroupe des représentants d'administrations publiques, d'institutions techniques, ainsi que des représentants d'agriculteurs et de consommateurs. Il lui est attaché un secrétariat permanent qui s'occupe de la préparation des réunions et de la rédaction des procès-verbaux. Les sous-comités spécialisés sont composés d'acteurs publics (des

1. La loi d'orientation agricole $n^{\circ} 08-16$ du 3 août 2008.

2. Le décret exécutif n 13-206 du 7 juillet 2013. représentants du ministère de l'Agriculture et du Développement rural et de ses services déconcentrés), d'acteurs privés (représentants d'associations de producteurs) et d'acteurs associatifs (représentants d'associations de protection des consommateurs) pour l'élaboration des cahiers des charges. Le sous-comité sollicite l'expertise des parties compétentes concernées par la connaissance du produit soumis à la labellisation (technicien, ingénieur, chercheur spécialiste du produit en question). La validation et la conformité des produits aux spécifications des cahiers des charges pour l'octroi des signes sont assurées par un organisme de certification indépendant, habilité par l'organisme algérien d'accréditation.

\section{La gouvernance territoriale comme nouvelle modalité de l'action publique}

L'action publique s'appuie de plus en plus sur des référentiels puisés dans les modèles de la nouvelle gouvernance : cette nouvelle modalité d'intervention intègre le principe d'une participation plus large d'acteurs dans la définition des projets de développement des territoires (Lorrain, 2005). Cette participation suscite une dynamique de concertation et de coopération qui est susceptible d'aboutir à des normes communes (Angeon et Laurol, 2006). Agissant sur la réalité et les problématiques au niveau du territoire, les instruments qui sont mis en place véhiculent une construction collective de connaissances et répondent à des enjeux territorialisés (Lascoumes et Simard, 2011). C'est dans cette voie qu'en Algérie la politique du renouveau agricole est mise en place en 2009.

Les nouveaux enjeux de cette politique publique ont suscité un renouvellement de l'action publique en matière de gouvernance de l'agriculture et des territoires ruraux. Ils ont initié des instruments et dispositifs prenant en compte l'approche participative dans la valorisation et la promotion de la qualité liée à l'origine. 
L'indication géographique, en tant qu'instrument mis en œuvre dans le cadre de la politique du renouveau agricole, marque une rupture avec les modes opératoires utilisés par le passé. Cet instrument se traduit par l'introduction d'éléments nouveaux comme la valorisation du terroir (savoir, savoir-faire et produit) et la sollicitation des acteurs locaux. Le dispositif d'indication géographique regroupe en effet des logiques à la fois organisationnelle, patrimoniale, commerciale et territoriale. Il correspond à cet égard aux différentes dimensions identifiées par Sylvander et al. (2006) à savoir : (a) l'organisation de la concurrence, (b) la maîtrise des marchés agricoles, (c) le développement local, rural et territorial, (d) le patrimoine (naturel et culturel) et la conservation des ressources. L'IG contribue en ce sens à réaliser et fixer la valeur ajoutée sur le territoire (Mollard et Pecqueur, 2007), à mobiliser les acteurs locaux autour d'une activité locale en assurant des revenus viables et durables et à créer de l'activité, afin de fixer les populations locales et redynamiser le territoire (Colletis et Pecqueur, 1993). Elle engendre de la concurrence loyale entre les producteurs et les autres acteurs impliqués dans la démarche, et assure au consommateur une qualité spécifique du produit en garantissant son origine.

\section{La gouvernance territoriale comme un processus d'apprentissage}

La gouvernance territoriale recouvre l'ensemble des situations de coopération et de construction d'action collective dans les organisations non hiérarchiques où l'État joue le rôle de précurseur à travers la mise en place d'instruments et de dispositifs réglementaires. La gouvernance territoriale est considérée comme une modalité ou un mécanisme permettant une coordination efficace d'acteurs ou d'entités organisationnelles sur un territoire (Michaux, 2011). Associant aux acteurs publics (État, collectivités territoriales, etc.), des acteurs privés, appartenant au monde de l'entreprise et/ou à la société civile comprise au sens large (associations, organisations non gouvernementales, syndicats, etc.) (Baron, 2003).

La gouvernance territoriale est dès lors synonyme d'implication de la société civile dans la prise de décision et des acteurs locaux qui sont sollicités pour répondre collectivement aux problématiques qui les concernent. Ces acteurs doivent être en mesure d'institutionnaliser les principes de la gouvernance à travers l'acquisition de nouvelles connaissances permettant l'action collective (Rey-Valette et al., 2011). Cette gouvernance permet de nouvelles formes de coordination et de décision au niveau du territoire, et met donc en jeu de nouvelles pratiques qui supposent des apprentissages. Dans ce sens, Chia et al. (2008b) expliquent que la gouvernance favorise l'action collective à travers les processus d'apprentissage. Elle permet la production d'un langage, d'un projet et de règles communes permettant ainsi aux acteurs de mettre en place des processus d'apprentissage qui favorisent l'action collective. Cette gouvernance peut être analysée à travers les stratégies d'acteurs, la capacité locale d'adaptation et les processus d'apprentissage (Gilly et Pecqueur, 2002).

La littérature sur le sujet souligne le lien entre gouvernance et apprentissage. Ce lien est évoqué soit de façon explicite (Chia et al., 2008b ; Gilly et Pecqueur, 2002), soit de façon implicite quand les auteurs parlent de nouvelles pratiques permettant l'acquisition de connaissances, de capacités et d'aptitudes (Leloup et al., 2005). Les apprentissages évoqués sont les apprentissages collectifs qui influencent les formes de coordination, les relations de coopération et la dynamique collective (Gilly et Pecqueur, 2002 ; Chia et al., 2008b). Ces apprentissages organisationnels ont un rôle 
dans l'action collective, la mutualisation et la mise en place de réseaux (Rey-Valette et al., 2011).

Les apprentissages en tant que supports à la création de nouvelles connaissances, de nouveaux savoir-faire et de nouvelles compétences des acteurs sont essentiels pour comprendre le processus de gouvernance territoriale (Rey-Valette et al., 2011). Ces apprentissages, sous l'impulsion des initiatives locales ou en réponse aux dispositifs mis en œuvre par les pouvoirs publics, permettent aux territoires de se différencier les uns par rapport aux autres, mais également de maintenir des processus de développement (Torre et Wallet, 2012).

Plusieurs auteurs appréhendent les processus de gouvernance des territoires en se focalisant sur les instruments et les dispositifs d'action (Lardon et al., 2008). L'instrument est considéré comme marqueur concret de l'action publique et vecteur de changement (Lascoumes et Le Galès, 2005 ; Lascoumes et Simard, 2011). En plus d'être porteurs de changement, les instruments jouent un rôle actif dans la construction, la stimulation et la capitalisation de l'apprentissage, particulièrement quand il est question de l'émergence de nouveaux savoirs et de leurs apprentissages. En plus de leurs rôles de contenant de savoir et d'interprétation d'action, ils peuvent très bien entraîner des dynamiques d'action collective, voire les modifier (Hatchuel et Weil, 1992). En ce sens, les instruments déclenchent des apprentissages et notamment des apprentissages de gouvernance, dans lesquels il est primordial qu'il y ait partage de représentations mentales, de valeurs communes, partage d'une vision commune et établissement de la confiance entre les différentes parties prenantes (Vitry, 2014). La confiance contribue à renforcer le comportement coopératif. Des auteurs comme Duguid et al. (2007) considèrent que la capacité à communiquer, à coopérer, et à travailler en équipe, sont des éléments centraux de la gouvernance territoriale.

Ce sont ces apprentissages collectifs qui nous permettent d'appréhender le processus de construction de la gouvernance territoriale.

\section{Méthodologie}

\section{Grille d'analyse des apprentissages}

Nous avons analysé les apprentissages produits dans un contexte de gouvernance territoriale sur la base du travail de Vitry (2014). Dans la présente recherche, nous proposons de retenir les variables d'apprentissage en fonction de leur relation avec les processus de gouvernance territoriale (tableau 1).

Ces quatre types d'apprentissages sont ceux qui permettent aux acteurs du

Tableau 1. Type d'apprentissage produit par une situation de gouvernance

\begin{tabular}{|c|c|c|}
\hline Auteur & Variable d'apprentissage & Indicateur d'apprentissage \\
\hline $\begin{array}{l}\text { Sol et al. (2013); } \\
\text { McAllister (1995) }\end{array}$ & Construction de la confiance & $\begin{array}{l}\text { Augmentation de la participation, } \\
\text { renforcement du comportement } \\
\text { coopératif }\end{array}$ \\
\hline $\begin{array}{l}\text { Senge (2006) ; } \\
\text { Dieleman (2013) }\end{array}$ & $\begin{array}{l}\text { Processus qui modifient les modèles } \\
\text { mentaux partagés }\end{array}$ & $\begin{array}{l}\text { Reconnaissance des erreurs du passé, } \\
\text { le besoin d'agir collectivement }\end{array}$ \\
\hline Rist et al. (2007) & $\begin{array}{l}\text { Définition d'une vision commune } \\
\text { du territoire. }\end{array}$ & $\begin{array}{l}\text { Capacité des acteurs à rendre compte } \\
\text { des savoirs tacites }\end{array}$ \\
\hline Duguid et al. (2007) & Capacité d'organisation & $\begin{array}{l}\text { Collaboration d'acteurs de différentes } \\
\text { catégories et de différents niveaux }\end{array}$ \\
\hline
\end{tabular}


territoire d'apprendre à coopérer et à se coordonner. Les variables d'apprentissage permettent d'appréhender l'aptitude des acteurs à partager une vision commune du territoire. Le travail collaboratif associant les acteurs privés et associatifs permet l'échange, l'expression et la construction d'un langage commun pour des acteurs qui ne partagent pas forcément les mêmes intérêts initiaux. Cette nouvelle forme de participation favorise également l'émergence d'un cadre mental collectif et de représentations communes.

Ces variables d'apprentissages sont mobilisées dans ce travail comme outil de lecture et d'analyse. L'application de cette grille d'analyse à un contexte autre que celui pour lequel elle a été construite permet d'illustrer le caractère opérationnel et exploratoire de cette grille.

\section{2. Étude de cas : l'indication géographique "figue sèche de Béni Maouche "}

Afin de déterminer les apprentissages résultant de la mise en place de l'indication géographique, nous optons pour une étude de cas qui vise à analyser les coordinations entre les acteurs parties prenantes dans le processus de mise en place de l'indication géographique «figue sèche de Béni Maouche»(wilaya de Bejaïa).
La méthodologie adoptée dans cette recherche vise à identifier non seulement les acteurs clés impliqués, mais aussi les interactions induites dans la mise en place du système national de labellisation.

Pour ce faire, après l'analyse du processus de mise en place du système national de labellisation réglementaire sur les volets, structurel et organisationnel, et l'identification des différentes parties prenantes (tableau 2), nous avons mené des entretiens semi-directifs auprès d'acteurs choisis selon leur implication dans le processus de labellisation. Nous avons mené des entretiens avec les responsables du dossier de labellisation qui nous ont permis l'identification des catégories d'acteurs impliqués dans le processus de labellisation (tableau 2). À la suite de cette identification, 26 entretiens ont été menés auprès d'un échantillon d'acteurs de chaque catégorie. Ils ont duré entre une heure trente et deux heures, et se sont étalés sur les années 2015 à 2017. L'enquête a donc permis de suivre le développement sur le terrain du projet de labellisation qui a démarré en avril 2014.

Les entretiens ont été complétés par une observation participante, notamment lors de l'atelier scientifique et technique et l'atelier de restitution du projet. Tous les

\section{Tableau 2. Type et catégorie d'acteurs impliqués dans le processus de labellisation}

\begin{tabular}{|c|c|c|}
\hline Partie prenante & Types d'acteurs & Catégories d'acteurs enquêtés \\
\hline Acteurs privés & Producteurs locaux & $\begin{array}{l}7 \text { producteurs de Béni Maouche; } \\
4 \text { producteurs des communes limitrophes }\end{array}$ \\
\hline Association & Associations des producteurs & $\begin{array}{l}\text { Président de l'association communale des figuiculteurs } \\
\text { de Béni Maouche ; secrétaire général de l'association }\end{array}$ \\
\hline \multirow[t]{3}{*}{ Acteurs publics } & Acteurs publics centralisés & $\begin{array}{l}3 \text { responsables ministériels; } \\
3 \text { experts de l'Union européenne }\end{array}$ \\
\hline & Acteurs publics locaux & $\begin{array}{l}2 \text { représentants de la Direction des services agricoles } \\
\text { (DSA) }\end{array}$ \\
\hline & Instituts techniques & $\begin{array}{l}4 \text { représentants (Institut national de recherche } \\
\text { agronomique et Institut technique d'arboriculture } \\
\text { fruitière et de la vigne) }\end{array}$ \\
\hline
\end{tabular}


entretiens ont été enregistrés et retranscrits et ont fait l'objet d'une analyse thématique selon les recommandations de la littérature (Yin, 2009). Nous nous sommes référés aux thèmes: participation, coordination concertation, coopération, confiance et vision commune.

L'indication «figue sèche de Béni Maouche » est un signe de qualité mis en place dans le cadre du dispositif de reconnaissance de la qualité des produits agricoles et agroalimentaires. L'IG existe officiellement depuis 2016. La figue sèche de Béni Maouche a été sélectionnée pour son potentiel qualitatif et sa réputation. Le dispositif organisationnel préalablement existant (association de producteurs) a pu servir de base pour la construction de l'indication. Le périmètre de l'IG regroupe la commune de Béni Maouche et plusieurs communes voisines de montagne de la rive sud-est de la Soummam.

Notre étude a porté sur Béni Maouche, une région montagneuse située dans la wilaya de Bejaïa sur la rive sud de la Soummam. Béni Maouche est composé de 28 villages de plus de 20000 habitants répartis sur une superficie totale de près de $95 \mathrm{~km}^{2}$. C'est une région agricole et rurale où l'oléiculture et la figuiculture correspondent à $96 \%$ des plantations. Le figuier y représente $24 \%$ de la surface agricole soit près de 1000 ha (DSA, 2016).

\section{Principaux résultats}

Deux parties structurent nos résultats : une typologie des acteurs, qui précise les rôles et les modalités de concertation lors du processus de mise en place de l'IG, et les phases d'apprentissage identifiées lors de ce processus de construction collective.

\section{La mise en œuvre de l'indication géographique : une multitude d'acteurs}

Nous avons répertorié sept types d'acteurs que nous avons classés en quatre catégories
: les acteurs publics, les experts, les bénéficiaires et les animateurs, selon leur rôle dans le dispositif de reconnaissance de la qualité (voir tableau 3, annexe).

Les premiers contribuent à produire un cadre de référence réglementaire pour la mise en place de l'IG. Il s'agit :

- des acteurs publics centraux : les ministères du Commerce et de la Culture, le ministère de l'Agriculture, la Chambre nationale d'agriculture, et des organismes de certification : l'Institut national de propriété intellectuelle et l'organisme d'accréditation,

- des acteurs publics locaux : les directions déconcentrées du ministère de l'Agriculture, la direction des services agricoles, la Chambre d'agriculture de wilaya.

La seconde catégorie d'acteurs contribue à accompagner et former les acteurs publics et les professionnels. Cette catégorie comprend :

- Les experts de l'Union européenne,

- Les ingénieurs et techniciens des instituts techniques et de recherche du domaine agricole Institut national de recherche agronomique (INRA) et Institut technique d'arboriculture fruitière et de la vigne (ITAFV).

La troisième catégorie est composée des demandeurs et des bénéficiaires de l'IG, soit :

- des leaders professionnels : un groupe de producteurs de Béni Maouche qui œuvre depuis les années 1990 à promouvoir la figue locale. Il a participé à des manifestations nationales et internationales et organise dès 1996 la fête de la figue sèche au niveau de Béni Maouche. Envisageant l'opportunité de qualifier la figue sèche et leur territoire à travers un label, il se constitue dès 2006 en association (l'Association communale des figuiculteurs de Béni Maouche), offrant ainsi un cadre pour 
les actions collectives à une échelle territoriale,

- des producteurs adhérents à l'association communale des figuiculteurs,

- des producteurs de Béni Maouche,

- des producteurs des communes limitrophes.

Enfin, la quatrième catégorie, constituée par les animateurs, est un type hybride composé d'experts nationaux et de leaders professionnels qui participent à la sensibilisation des producteurs locaux, aux discussions entre les différents types d'acteurs, en assurant le rôle de médiation.

\section{La mise en œuvre}

de l'indication géographique :

\section{quatre phases d'apprentissage}

Nous avons identifié quatre phases d'apprentissage lors de la mise en place de l'IG : la construction d'un cadre collectif concernant la délimitation de l'aire géographique, le partage d'une vision commune dans l'élaboration du cahier des charges la construction de la confiance qui se traduit par l'augmentation de la participation et la capacité d'organisation à travers la création de différentes instances de régulation.

\section{La construction de la confiance : augmentation de la participation}

Le processus de mise en place du dispositif de reconnaissance de la qualité liée au territoire a été conçu en quatre étapes sollicitant la participation des différentes catégories d'acteurs. Selon les enjeux visés et le type d'acteur participant, les modalités de rencontres ont varié.

La construction du cadre réglementaire du dispositif s'est déroulée à travers des réunions et des ateliers de formation (56 au total) regroupant les acteurs publics centraux et les experts de l'Union européenne. Le processus décisionnel du Comité national de labellisation a pris forme à travers des ateliers de formation et des visites d'étude (54 au total), organisées entre le ministère de l'Agriculture, les experts nationaux et les experts européens.

La sensibilisation des acteurs locaux sur le rôle et l'intérêt des indications géographiques et appellations d'origine s'est faite quant à elle dans le cadre d'ateliers de formation et de journées d'information. Une centaine de rencontres ont réuni le ministère de l'Agriculture, la Chambre nationale d'agriculture, les experts et les leaders professionnels. La caractérisation de la figue sèche et de son terroir a nécessité pas moins de 44 rencontres déclinées sous forme de journées de formation et de journées d'étude organisées au niveau national et local, réunissant le ministère de l'Agriculture, la direction des services agricoles, la Chambre de l'agriculture de la wilaya, les experts, les demandeurs et les bénéficiaires.

Ces rencontres ont regroupé différents types d'acteurs qui, jusque-là, n'avaient pas l'habitude de travailler ensemble. L'institutionnalisation de ces rencontres, qui associent l'ensemble des parties prenantes, a été cruciale. Un cadre formel d'échange entre les acteurs a été établi et ces rencontres (journées d'étude, ateliers de formation et d'information) ont constitué des espaces collectifs, où l'expression et le débat sont très ouverts. Les professionnels et les producteurs peuvent faire part dans ce cadre de leurs préoccupations et de leurs problématiques. Cette dynamique contraste avec l'échec des coopératives agricoles de services créées dans les années 1960 à l'initiative des pouvoirs publics : celles-ci ont engendré une situation de méfiance vis-à-vis de l'action collective, ce qui a entraîné une faible participation des producteurs locaux.

L'institutionnalisation de ces rencontres a permis de rétablir la confiance des producteurs dans l'action collective. L'association communale des producteurs a vu, le nombre de ses sociétaires passer de 125 (avant le début de la mise en place de l'IG) à 342. Cette augmentation des 
effectifs traduit le regain de confiance des producteurs. Le dispositif de l'indication géographique semble avoir produit des situations d'enrôlement dans le sens où les producteurs impliqués et convaincus du bien-fondé de l'action collective ont promu celle-ci en motivant d'autres producteurs à y participer.

Ces rencontres propices aux échanges et aux dialogues entre les différentes catégories d'acteurs laissent une large place à la construction d'une vision commune de l'IG et d'un langage commun entre les experts, les professionnels et acteurs publics. Ceci se révèle important pour le futur de l'indication.

\section{La construction d'un cadre collectif :} délimitation de l'aire géographique

L'aire géographique de production de la figue sèche de Béni Maouche qui couvre 22 communes réparties entre deux wilayas (Bejaïa et Sétif) a été définie de manière collective. Sa délimitation est issue d'un processus de dialogue et de négociations entre les experts, les acteurs publics et les producteurs (groupe leader et producteurs de Béni Maouche), au cours desquels se sont révélés les intérêts, objectifs et motivations de chaque acteur.

Pour étendre le développement à toute la région, les acteurs publics espéraient un territoire de production élargi, contrairement aux producteurs de la commune qui préféraient une aire de production limitée, car la dénomination de l'IG portait le nom de leur commune. Les producteurs de Béni Maouche pensaient restreindre l'aire de l'indication à leur commune pour être les seuls bénéficiaires. Ils ont refusé la participation des producteurs des communes voisines aux rencontres qui se déroulaient dans le cadre de la définition de la figue et de son territoire. La conduite de consultation avec les producteurs de Béni Maouche a révélé que les facteurs identitaires et le sentiment d'appartenance tiennent une place importante dans la représentation que les producteurs de Béni Maouche se font de l'IG et du territoire de production. Dès lors, le défi majeur pour les acteurs publics et les experts est d'amener les producteurs de Béni Maouche à adopter une vision territoriale élargie.

Dans cette perspective, des journées d'étude et des ateliers d'information ont été organisés par les acteurs publics centraux et les experts pour informer et discuter avec les producteurs du bien-fondé d'un territoire élargi. Elles ont permis de rappeler que les critères déterminants la délimitation de l'aire de production ne se cantonnent pas à la composante «territoire/ identité », mais reposent en grande partie sur l'identification de critères scientifiques (les conditions agropédoclimatiques, l'homogénéité du type de système de production et la variété). Les discussions avec les experts et les acteurs publics locaux ont fait infléchir l'opposition des producteurs de Béni Maouche qui ont accepté de participer à la délimitation d'un territoire de production élargi de l'indication.

Le résultat de ces discussions consiste en une définition collective, élargie et inclusive du territoire de production, traduisant le passage d'une perception limitée à une perception élargie du territoire. Ceci constitue une rupture avec la représentation antérieure de l'IG (authentification de provenance) et témoigne du partage d'une représentation commune, dans laquelle l'IG est perçue comme un signe de reconnaissance de la qualité d'un produit dont la typicité est en lien avec le territoire où il est produit. Cette nouvelle interprétation est une étape nécessaire dans le processus de construction de la qualité territoriale des produits.

\section{Une vision commune dans \\ la construction du cahier des charges}

Le cahier des charges est le résultat d'un processus de construction commune des spécificités du produit entre différentes parties prenantes aux visions parfois divergentes. Les producteurs locaux 
(association communale des producteurs, le groupe animateurs, les producteurs de Béni Maouche et ceux des communes voisines) visent une définition du produit qui sauvegarde la qualité et la réputation de la figue. Les experts ont pour objectif de formaliser les savoir-faire locaux et de les normaliser. Enfin, les acteurs publics locaux souhaitent instituer des règles opérationnelles pour le contrôle de conformité.

Le cadre d'interaction fourni par la délimitation de l'aire géographique apparaît alors comme la base de consensus. En effet, la participation des producteurs des communes voisines aux discussions sur le cahier des charges montre la convergence des stratégies individuelles et de la stratégie collective. Le mode de production, les pratiques culturales, le stade de récolte et le support de séchage sont discutés avec les producteurs et traduits en normes par les experts. Les producteurs ont dû renoncer à certaines pratiques et adopter de nouvelles règles construites collectivement. Pour consolider la réputation et améliorer la qualité, les producteurs locaux ont accepté et adopté de nouvelles règles de séchage et de conservation du fruit, correspondant à de bonnes pratiques issues des recommandations des experts. Les pratiques autorisées ou proscrites sont le résultat de concertation entre les producteurs et les experts.

La construction du cahier des charges traduit la transcription des pratiques et des usages qui définissent la figue sèche de Béni Maouche en une série de normes et de consignes qui conditionnent la production, la transformation et la conservation. En ce sens, l'établissement de ces règles qui spécifient le produit marque le passage de connaissances tacites (savoir et savoir-faire des producteurs) à une série de connaissances explicites, de techniques et d'obligations qui encadrent la production et la conservation de la figue de Béni Maouche. Ce passage à un langage formel transmissible permet un accord des différentes parties prenantes sur une définition commune de ce qu' est la figue sèche de Béni Maouche.

\section{Développement des capacités d'organisation et émergence de l'action collective}

Au-delà de ces espaces de concertation sur la délimitation de l'aire de production et la définition du cahier des charges, de véritables formes d'action collective ont été mises en place. Nous distinguons celles qui ont émergé de la collaboration de participants de même type (figuiculteurs dans le cadre de l'association) de celles qui réunissent des acteurs de différents types, comme dans le cas du Comité de labellisation ou le groupe animateur. En effet, la mise en place de l'IG a conduit à l'émergence d'une action collective au niveau local, voire au-delà. Bien que l'acteur public joue un rôle important de prescripteur du dispositif de reconnaissance de la qualité, l'élaboration de l'indication a fait intervenir une multitude d'acteurs, lesquels, malgré des intérêts et des visions différentes, ont adhéré au projet de labellisation. Ils ont apporté une dynamique de coopération et de concertation établie à travers des rencontres et des discussions.

\section{La création de l'association}

des producteurs : une organisation de professionnels

La gestion, la promotion et la défense de l'IG ont été initialement prises en charge par l'association communale des producteurs. Cependant, cette association est une association agricole où les emballeurs et les conditionneurs ne sont pas représentés et dont les activités sont confinées à la commune de Béni Maouche. La solution a été la création d'une nouvelle association (Association des producteurs de figues de la wilaya de Bejaïa-APFWB) avec une forte représentativité de tous les professionnels (producteurs, conditionneurs et emballeurs) et dont les actions 
dépassent les frontières de la commune pour atteindre toute la wilaya. La création de cette association correspond ainsi à une forme d'action collective qui témoigne de l'émergence de capacités d'organisation. La création de l'association des producteurs de la wilaya de Bejaïa traduit un nouveau comportement adopté par les acteurs locaux pour s'adapter à une transformation de l'environnement. Nous pouvons dire qu'il s'agit d'une nouvelle action collective organisationnelle par interaction et socialisation au sens de Nonaka (1994).

\section{Création du Comité national de \\ labellisation : une coordination institutionnelle}

La création du Comité national de labellisation est un espace de coordination et de collaboration entre différentes catégories d'acteurs, de nature publique et associative, qui participent aux différentes réunions de façon régulière et travaillent ensemble pour l'élaboration et le contrôle du processus de labellisation. Nous constatons cependant une forte représentativité d'acteurs publics avec notamment des représentants de différents ministères en plus du ministère de l'Agriculture, porteur du projet de labellisation.

Le Comité national de labellisation, de par ses missions d'élaboration, de suivi et de contrôle, est impliqué à toutes les étapes du processus de reconnaissance de la qualité. Il joue le rôle de structure de pilotage, d'incitation, de contrôle et d'arbitrage du système de reconnaissance de la qualité. $\mathrm{Au}$ regard de sa composition (les acteurs publics traditionnels, les acteurs privés et la société civile), le Comité de labellisation est une structure de gouvernance hybride, telle que définie par Raynaud et Sauvée (2000).

\section{Émergence du Comité local d'animation : l'amorce d'une capacité organisation}

Au niveau local, un groupe d' animateurs est constitué, servant de relais permanent entre les acteurs centraux experts nationaux et européens et les acteurs locaux. Il est composé d'un élu local, de deux ingénieurs de la direction des services agricoles de la wilaya de Bejaïa, de deux techniciens de l'institut technique de l'arboriculture et du secrétaire général de la chambre d'agriculture de la wilaya. Un groupe d'acteurs très hétérogène, mais qui a travaillé pour être parfois une structure de communication (quand il a été question de vulgarisation des démarches qualitatives, de l'intérêt de l'opportunité de labelliser auprès des producteurs pour obtenir leur adhésion), ou d'information (quand il fallait mettre au courant les producteurs, des intentions ou des recommandations prises par les experts nationaux ou européens). Ce groupe animateur a construit un espace de réflexion et de dialogue informatif pour les producteurs locaux et consultatif pour les acteurs publics centraux, soit une forme d'interface entre les acteurs publics et les producteurs locaux.

La formation de ce groupe témoigne du développement d'une capacité d'organisation chez les acteurs locaux qui se traduit aussi bien dans l'organisation de rencontres, que dans la discussion et le dialogue sur les questions d'établissements des critères techniques du produit ou parfois même de négociation et de concertation quand il a été question de définir les contours de l'aire géographique de production. Ce groupe s'est distingué dans l'animation du débat offrant ainsi aux acteurs locaux un espace de discussions, de négociation et de concertation qui a permis par la suite la prise de décisions communes.

La mise en place de l'IG a poussé les acteurs à adopter des modalités d'organisation collective, et cela à plusieurs niveaux. En somme, ces organisations composées d'acteurs de différentes catégories et de différents niveaux, qui au-delà de leur hétérogénéité, ont contribué à définir des actions communes dans le cadre de la mise en place de l'IG. La formation de ces organisations traduit le développement de 
capacités réelles d'organisation avec des formes variables de participation aux débats et à la décision.

$$
\begin{gathered}
* \\
* \quad *
\end{gathered}
$$

L'objectif de cet article était de déterminer comment un dispositif, mis en place dans une démarche descendante par l'État central, a suscité une participation plus large d'acteurs locaux. Au-delà de leur hétérogénéité, les différentes catégories d'acteurs se situant à différentes échelles se sont coordonnées autour d'un objectif commun. L'action collective locale s'est construite à travers un espace de discussion, de négociation et de concertation.

Ce travail avait pour ambition d'analyser la construction d'une gouvernance territoriale par les apprentissages liés à la mise en place d'un dispositif réglementaire de reconnaissance de la qualité. L'analyse de la gouvernance territoriale vue à travers le prisme de l'apprentissage a permis une identification des principaux moteurs des changements organisationnels produits pour la reconnaissance de la qualité territoriale d'un produit. En effet, les démarches participatives, ainsi que les relations horizontales basées sur la négociation et la concertation, mises en œuvre dans le cadre du système national de labellisation, ont incité au changement. Elles ont fait émerger des comportements et des compétences en matière d'action collective de la part de plusieurs acteurs investis dans la démarche de labellisation et dans le processus de prise de décision.

L'analyse des évolutions institutionnelles et organisationnelles produites dans le cadre de la mise en place de ce dispositif révèle différentes situations d'apprentissage. En effet, l'indication géographique a permis une structuration de l'action collective (Gilly et Pecqueur, 2002) : la constitution du Comité de labellisation, de l'association des producteurs de Bejaïa et du Comité d'animation montrent le renforcement de capacités d'organisation. La définition du territoire de production a abouti à l'abandon de la première vision de l'IG et l'adoption de nouvelles règles construites collectivement, signe de l'instauration d'un code commun partagé par l'ensemble des acteurs (Chia et al., 2008b). En ce sens, la construction du cahier des charges témoigne de l'établissement d'une vision commune. Elle met en évidence différents niveaux de coordination entre les parties prenantes (Leloup et al., 2005), traduisant la transcription de ces savoirs et savoir-faire (connaissance tacite) en un référentiel du processus productif (connaissance explicite). La mise en place de l'IG s'est déroulée sur plus d'une centaine de rencontres entre les différentes parties prenantes, créant une dynamique d'échange et une institutionnalisation des rencontres qui a conduit à l'établissement ou le renforcement du climat de confiance. Toutes ces situations sont révélatrices d'un apprentissage constructif de la gouvernance territoriale.

Ces résultats apportent des compléments à la littérature sur le rôle de l'apprentissage dans la mise en œuvre de l'action collective pour la gouvernance territoriale. Par rapport aux travaux de Rey-Valette et Mathé (2012) qui considèrent l'apprentissage comme un moyen d'évaluation des politiques publiques territoriales, nous montrons que ces politiques peuvent être l'occasion de produire des apprentissages de l'action collective.

L'observation du dispositif de reconnaissance de la qualité liée au territoire a permis de produire une analyse critique de sa mise en œuvre et de cerner les apprentissages acquis lors de la mise en place de l'IG certes, mais suggère par ailleurs de nouvelles interrogations sur les conditions de pérennisation des modalités de gouvernance territoriale et sur les modalités d'appropriation par les acteurs des nouveaux instruments basés sur le principe de participation et de partenariat. 


\section{RÉFÉRENCES BIBLIOGRAPHIQUES}

Angeon V., Laurol S. (2006). Les pratiques de sociabilité et de solidarité locales : contribution aux enjeux de développement territorial. Espaces et sociétés, vol. 127, $\mathrm{n}^{\circ} 4$, pp. 13-31.

Baron C. (2003). La gouvernance : débat autour d'un concept polysémique. Droit et Société, $n^{\circ} 54$, pp. 329-351.

Chia E., Dulcire M., Piraux M. (2008a). Le développement d'une agriculture durable a$\mathrm{t}$-il besoin de nouveaux apprentissages? Les leçons tirées d'une recherche en milieu insulaire (Réunion). Études caribéennes, ${ }^{\circ} 11$, décembre, mis en ligne le 15 décembre 2008.

Chia E., Torre A., Rey-Valette H. (2008b). Conclusion : vers une «technologie» de la gouvernance territoriale ! Plaidoyer pour un programme de recherche sur les instruments et dispositifs de la gouvernance des territoires. Norois, vol. 209, $\mathrm{n}^{\circ}$ 4, pp. 167-177.

Colletis G., Pecqueur B. (1993). Intégration des espaces et quasi-intégration des firmes : vers de nouvelles logiques productives. Revue d'économie régionale et urbaine, $\mathrm{n}^{\circ} 3$, pp. 490-507.

Dieleman H. (2013). Organizational learning for resilient cities, through realizing ecocultural innovations. Journal of Cleaner Production, $\mathrm{n}^{\circ}$ 50, pp. 171-180.

Duguid F., $\quad$ Mündel K., $\quad$ Schugurensky D. (2007). Volunteer work, informal learning, and the quest for sustainable communities in Canada. Canadian Journal for the Study of Adult Education, vol. 20, n ${ }^{\circ}$, pp. 41-56.

DSA (2016). Rapport sur la production agricole.

Gilly J.-P., Wallet F. (2001). Forms of proximity, local governance and the dynamics of local economy spaces: the case of industrial conversion process. International Journal of Urban and Regional Research, vol. 25, $\mathrm{n}^{\circ} 3$, septembre, pp. 553-570.

Gilly J.-P., Pecqueur B. (2002). La dimension locale de la régulation. In Robert Boyer, Yves Saillard (dir.), Théorie de la régulation : l'état des savoirs (2 éd., pp. 304-312). Paris, La Découverte, coll. « Recherches ».
Hatchuel A., Weil B. (1992). L'expert et le système : gestion des savoirs et métamorphose des acteurs dans l'entreprise industrielle; suivi de quatre histoires de systèmes-experts. Economica, 264 p.

Lardon S., Chia E., Rey-Valette H. (2008). Introduction : dispositifs et outils de gouvernance territoriale. Norois, $\mathrm{n}^{\circ}$ 209, pp. 7-13.

Lascoumes P., Le Galès P. (2005). Gouverner par les instruments. Paris, Presses de Sciences Po (P.F.N.S.P.), 370 p.

Lascoumes P., Simard L. (2011). L'action publique au prisme de ses instruments : introduction. Revue française de science politique, vol. 61, pp. 5-22.

Leloup F., Moyart L., Pecqueur B. (2005). La gouvernance territoriale comme nouveau mode de coordination territoriale. Géographie, économie, société, vol. 7, $\mathrm{n}^{\circ} 4$, pp. 321-332.

Lorrain D. (2005). Les pilotes invisibles de l'action publique: Le désarroi du politique? In Gouverner par les instruments, Paris, Presses de Sciences Po (P.F.N.S.P.), pp. 163-197.

McAllister D J. (1995). Affect and cognitionbased trust as foundations for interpersonal cooperation in organizations. The Academy of Management Journal, vol. 38, $\mathrm{n}^{\circ} 1$, pp. 24-59.

Michaux V. (2011). Les déterminants de la performance des gouvernances territoriales. Le cas des stratégies concertées de développement durable des territoires. Revue française de gestion, vol. 217, $\mathrm{n}^{\circ}$ 8, pp. 35-60.

Mollard A., Pecqueur B. (2007). De l'hypothèse au modèle du panier de biens et de services. Histoire succincte d'une recherche. Économie rurale, $\mathrm{n}^{\circ} 300$, pp. 110-114.

Nonaka I. (1994). A dynamic theory of organizational knowledge creation. Organization Science, vol. 5, $\mathrm{n}^{\circ}$ 1, pp. 14-37.

Raynaud E., Sauvée L. (2000). Signes collectifs de qualité et structures de gouvernance. Économie rurale, $\mathrm{n}^{\circ}$ 258, pp. 101-112.

Rey-Valette H., Pinto M., Maurel P., Chia E., Guihéneuf P.-Y., Michel L., Nougarèdes B., 
Soulard C., Jarrigue F., Guillemot S., Cunnac M., Mathé S., Barbe E., Ollagnon M., Delorme G., Prouhèze N., Laurent A., Suita L. A., Bertrand J., Dieudonné C., Morvan R., Champigraud A. (2011). Guide pour la mise en ceuvre de la gouvernance en appui au développement durable des territoires. Cemagref, CNRS, Geyser, Inra, SupAgro, Université Montpellier 1, $155 \mathrm{p}$.

Rey-Valette H., Mathé S. (2012). L'évaluation de la gouvernance territoriale. Enjeux et propositions méthodologiques. Revue d'économie régionale \& urbaine, décembre, $\mathrm{n}^{\circ}$ 5, pp. 783-804.

Rist S., Chidambaranathan M., Escobar C., Wiesmann U., Zimmermann A. (2007). Moving from sustainable management to sustainable governance of natural resources: The role of social learning processes in rural India, Bolivia and Mali. Journal of Rural Studies, vol. 23, n 1 , pp. 23-37.

Senge P. (2006). The fifth discipline: The art and practice of the learning organization. Random House Business (2nd ed.), 464 p.

Simoulin V. (2007). La gouvernance territoriale. Pratiques, discours et théories, LGDJ. Droit et société, pp. 15-32.
Sol J., Beers P. J., Wals A.E.J. (2013). Social learning in regional innovation networks: trust, commitment and reframing as emergent properties of interaction. Journal of Cleaner Production, $\mathrm{n}^{\circ}$ 49, pp. 35-43.

Sylvander B., Allaire G., Belletti G., Marescotti A., Thevenod-Mottet E., Barjolle D., Tregear A. (2006). Les dispositifs français et européens de protection de la qualité et de l'origine dans le contexte de l'OMC : justifications générales et contextes nationaux. Revue canadienne des sciences régionales, vol. 29, $\mathrm{n}^{\circ} 1$, pp. 43-54.

Torre A., Wallet F. (2012). Innovations et gouvernance des territoires ruraux. In Coudel E., Devautour H., Soulard C. T., Faure G., Hubert B., Apprendre à innover dans un monde incertain. Concevoir les futurs de l'agriculture et de l'alimentation, Versailles, Quae, 248 p.

Vitry C. (2014). Vers une théorie des apprentissages de gouvernance: une exploration en territoires périurbains. Thèse de doctorat sous la direction d'Eduardo CHIA. Montpellier SupAgro, 447 p.

Yin R. K. (2009). Case study research: design and methods. Thousand Oaks, Sage. 